\title{
Aspectos epidemiológicos da mortalidade infantil no município de Santarém-PA: uma abordagem retrospectiva
}

\author{
Epidemiological aspects of child mortality in the municipality of \\ Santarém-PA: a retrospective approach
}

\author{
1 Sheyla Mara Silva de Oliveira enfsheylauepastm@hotmail.com \\ 1 Franciane de Paula Fernandes \\ 1 Daniel Teixeira Oliveira \\ 1 João Vitor Ferreira Walfredo \\ 1 Francileno Sousa Rêgo
}

1 Universidade do Estado do Pará

\section{Resumo}

A mortalidade infantil constitui um dos índices mais importantes e sensíveis para avaliar os impactos de determinada doença ou causa na saúde de uma população. 0 presente estudo tem como objetivo estudar os aspectos epidemiológicos da mortalidade infantil no município de Santarém - Pará. A pesquisa foi de caráter quantitativo, descritivo, retrospectivo, documental e epidemiológico de delineamento longitudinal. 0 presente estudo analisou os 1.512 óbitos infantis ocorridos no município no período de 2007 a 2016. Os números máximo e mínimo atingidos, levando-se em consideração a proporção de óbitos infantis para cada mil nascidos vivos, foram, em 2009, com 163 óbitos para um total de 6.167 nascidos vivos e, em 2014, com 129 para um total de 6.595 nascidos vivos, respectivamente. Notadamente houve uma tendência de queda no índice de mortalidade infantil calculado, principalmente, do período de 2007 , com a taxa de mortalidade infantil de 24,82, e em 2014 , com a taxa de mortalidade de 19,56, que representa um decréscimo de $21,19 \%$. Tem-se um perfil de mães na primeira gestação $(87,00 \%)$, ao local da ocorrência, sendo majoritariamente o ambiente hospitalar $(95,00 \%)$, ao tipo de parto caracterizado como vaginal $(65,00 \%)$ e, por último, uma idade gestacional entre 37 a 41 semanas (33,00\%). O estudo nos remete o entendimento dos parâmetros de mortalidade no município, para que possamos auxiliar na implementação de políticas públicas para a região.

\section{Palavras- chave}

Epidemiologia. Mortalidade infantil. Saúde pública.

\section{Abstract}

Childs mortality is one the most important and sensitive indicators for assessing the impacts of a given disease or cause on the health of a population. This study aims to study the epidemiological aspets of child mortality in the municipality of Santarém-Pará. The research was quantitative, descriptive, retrospective, documental and of an epidemiological longitudinal design. The present study analyzed the 1,512 infant deaths that occurred in the municipality from 2007 to 2016 . The maximum and minimum numbers reached, taking into account the proportion of infant deaths per thousand live births, were in 2009, with 163 deaths for a total of 6,167 live births, and in 2014, with 129 for a total of 6,595 births, alive respectively. Notably, there was a downward trend in the child mortality rate calculated mainly from the 2007 period, with the child mortality rate of 24.82, to 2014, with the 19.56 mortality rate, which represents a decrease of $21,19 \%$. There is a profile of mothers in the first pregnancy equivalent to $87.00 \%$, the place of occurrence being mostly the hospital environment, $95.00 \%$, the type of delivery characterized as vaginal, being $65.00 \%$ and last, a gestacional age between 37 and 41 weeks, totaling $33.00 \%$. The study brings us the understanding of mortality parameters in the municipality so that we can assist in the implementation of public policies for the region.

\section{Keywords}

Epidemiology. Child mortality. Public health.

\section{Como você deve citar?}

OLIVEIRA, Sheyla Mara Silva de et al. Aspectos epidemiológicos da mortalidade infantil no município de santarém-pa: uma abordagem retrospectiva. Cadernos UniFOA, Volta Redonda, n. 42, p. 103-113, abril, 2020. 


\section{INTRODUÇÃO}

A redução de mortalidade infantil ainda é um dilema enfrentado pelos serviços de saúde como um todo, e não é um problema apenas de países como Brasil, mas, também, uma preocupação de cunho mundial atualmente. É tão relevante que faz parte das metas do Desenvolvimento do Milênio, uma responsabilidade assumida pelos países integrantes da Organização das Ações Unidas (ONU), com a finalidade de combater a pobreza, a fome, as doenças e o analfabetismo, por exemplo, galgando alcançar patamares mais dignos de vida para a sociedade, tendo em vista que a mortalidade infantil reflete as condições de vida da população (BRASIL, 2009).

Estipula-se que, ainda hoje, quatro milhões de recém-nascidos morram a cada ano no mundo. No entanto, um dado mais alarmante é que, aproximadamente, quase $70 \%$ dessas mortes sejam por causas evitáveis, como, por exemplo, uma inadequada prestação de assistência à mulher durante a gestação, no parto e ao recém-nascido (DE PAULA JÚNIOR et al., 2017). Não obstante, o Brasil se encaixa nessa realidade de forma positiva se tratando da redução da mortalidade infantil nas últimas décadas, porém, quando são levadas em consideração as macrorregiões, como a norte, esses índices ainda são significativos em comparação com as outras regiões e no contexto global (ROUQUAYROL, 2013).

Assim, com a perspectiva de abranger dados e informações de cunho quantitativo e qualitativo de óbitos no Brasil, o Sistema de Informação de Mortalidade (SIM) foi elaborado em 1975 como uma ferramenta essencial para a verificação da saúde no país. Desse modo, vários ramos da saúde usam o SIM para a obtenção de dados e informações, quando se tratando de mortalidade (BRASIL, 2017).

A mortalidade infantil no contexto nacional e global, quando analisada de forma geral, representa se os indicadores de saúde são positivos ou negativos e, além disso, leva em consideração outros fatores, como os sociais e econômicos. No entanto, a falta de conhecimento oficial sobre a morte pode representar um entrave para verificação adequada da saúde e, principalmente, para a real análise dos dados no momento de calcular os indicadores de mortalidade. Assim, verifica-se que a subnotificação de óbitos no Brasil é um problema a ser enfrentado, primordialmente em se tratando das regiões Norte e Nordeste. A omissão dos registros de óbitos em cartório compromete o real dimensionamento do problema e na identificação de ações adequadas para a diminuição da mortalidade (BRASIL, 2009).

O óbito de crianças menores de um ano é uma questão influenciada por diversas condições, entre elas, as de pré-natal, gravidez, história materna, conduta e doenças maternas, ruptura precoce de membrana, idade materna, procedimentos perinatais, mortalidade perinatal, condições e tipos de parto, síndrome da morte súbita, estado marital, intervalo entre partos, diferenças raciais materna e infantil, condições socioeconômicas, prematuridade, baixo peso ao nascer, más formações congênitas, mães portadoras do HIV e de outras doenças infectocontagiosas e outros (HARTZ, 1999).

Entre os anos de 2000 a 2010, no país, houve uma considerável diminuição do coeficiente de mortalidade infantil, passando de 21,3 óbitos por 1000 crianças nascidas vivas no primeiro ano para 13,9 óbitos por 1000 crianças nascidas vivas em 2010. Mesmo com essa perceptível redução, a mortalidade infantil permanece como uma preocupação para a saúde pública. A população mais pobre é a mais vulnerável, pois, por uma série de fatores, não consegue ajudar a sua prole a superar esse trecho inicial da vida. Em sua condição de pobreza, enfrenta dificuldades na vida pessoal e na comunitária familiar, pela falta de moradia, alimento, comunicação, transporte, trabalho e lazer (ROUQUAYROL, 2013).

É importante analisar o perfil da mortalidade infantil para a elaboração de medidas que possam vir a permitir o controle, que deve ser realizado desde a adoção de um amparo adequado à mulher durante 
a gestação e o parto até um acompanhamento pós-parto, principalmente das crianças consideradas de risco (VICTORA, 2001).

O presente estudo buscou analisar o perfil epidemiológico da mortalidade infantil no município de Santarém-PA.

\section{METODOLOGIA}

Este estudo é definido como um estudo descritivo, documental, retrospectivo de abordagem quantitativa. A coleta de dados foi feita no banco de dados do Sistema de Informação de Mortalidade (SIM) (DATASUS, 2019).

Foram incluídas informações contidas no banco de dados do Sistema de Informação de Mortalidade (SIM) do endereço eletrônico do DATA SUS ON LINE acerca dos óbitos infantis ocorridos no período de 2007 a 2016, no município de Santarém-PA; e coletadas ainda informações epidemiológicas das mães de crianças que vieram a óbito com até um ano de idade.

Foram excluídas informações acerca de óbitos de pessoas não ocorridos no município de SantarémPA, não ocorridos no período proposto e quaisquer dados não provenientes do Sistema de Informação de Mortalidade (SIM).

Para a elaboração do resultado desta pesquisa foram coletados os seguintes dados: sexo, Idade (mês/ dias), cor/raça, causa da morte de acordo com categoria CID-10, mês do óbito, peso ao nascer, duração da gestação, tipo de parto, local de ocorrência e informações sociodemográficas das mães de crianças que vieram a óbito com até um ano de idade.

Os resultados foram processados através de recursos da estatística descritiva, e quantificados a partir da frequência absoluta, mediante utilização do programa Excel (Microsoft for Windows - 2010), visando à construção de gráficos e tabelas. Para auxiliar a estatística, utilizamos o Programa Epilnfo versão 7.2 .

\section{RESULTADOS E DISCUSSÃO}

No presente estudo, verificou-se que o nível de escolaridade (Tabela 1) das mães em que os filhos evoluíram com óbito após o nascimento está mais acentuado na faixa de 8 a 11 anos, equivalente a, aproximadamente, 38\% ( $n=572)$, porém, tal dado entra em discordância com o estudo de França (2016), que verificou o maior índice de mortalidade infantil relacionado a mães com menos de oito anos de instrução. 
Tabela 1 - Escolaridade, cor e idade das mães de crianças que evoluíram com óbito de 2007 a 2016.

\begin{tabular}{|c|c|c|c|c|c|c|c|c|c|c|c|c|}
\hline & 2007 & 2008 & 2009 & 2010 & 2011 & 2012 & 2013 & 2014 & 2015 & 2016 & \multicolumn{2}{|c|}{ Total } \\
\hline \multicolumn{13}{|l|}{ Escolaridade } \\
\hline Nenhuma & 6 & 13 & 9 & 2 & 0 & 7 & 6 & 3 & 4 & 3 & 53 & $4 \%$ \\
\hline 1 a 3 anos & 16 & 16 & 9 & 5 & 11 & 13 & 8 & 6 & 8 & 9 & 101 & $7 \%$ \\
\hline 4 a 7 anos & 68 & 54 & 48 & 42 & 38 & 29 & 28 & 36 & 24 & 37 & 404 & $27 \%$ \\
\hline 8 a 11 anos & 49 & 47 & 65 & 66 & 56 & 57 & 45 & 48 & 67 & 72 & 572 & $38 \%$ \\
\hline 12 anos e mais & 21 & 15 & 16 & 13 & 9 & 17 & 13 & 11 & 13 & 14 & 142 & $9 \%$ \\
\hline Ignorado & 14 & 21 & 16 & 24 & 33 & 25 & 24 & 25 & 27 & 31 & 240 & $16 \%$ \\
\hline Total & 174 & 166 & 163 & 152 & 147 & 148 & 124 & 129 & 143 & 166 & 1512 & $100 \%$ \\
\hline \multicolumn{13}{|l|}{ Cor } \\
\hline Branca & 18 & 17 & 30 & 28 & 23 & 23 & 22 & 20 & 26 & 20 & 227 & $15 \%$ \\
\hline Preta & 1 & 1 & 3 & 2 & 2 & 0 & 0 & 2 & 0 & 0 & 11 & $1 \%$ \\
\hline Amarela & 0 & 1 & 1 & 0 & 0 & 0 & 0 & 0 & 0 & 0 & 2 & $0 \%$ \\
\hline Parda & 148 & 143 & 127 & 116 & 116 & 117 & 100 & 91 & 114 & 141 & 1213 & $80 \%$ \\
\hline Indígena & 1 & 2 & 1 & 0 & 3 & 0 & 0 & 1 & 2 & 4 & 14 & $1 \%$ \\
\hline Ignorado & 6 & 2 & 1 & 6 & 3 & 8 & 2 & 15 & 1 & 1 & 45 & $3 \%$ \\
\hline Total & 174 & 166 & 163 & 152 & 147 & 148 & 124 & 129 & 143 & 166 & 1512 & $100 \%$ \\
\hline \multicolumn{13}{|l|}{ Idade da Mãe } \\
\hline 10 a 14 anos & 8 & 4 & 2 & 1 & 3 & 3 & 2 & 4 & 1 & 1 & 29 & $2 \%$ \\
\hline 15 a 19 anos & 38 & 46 & 44 & 30 & 18 & 21 & 25 & 23 & 31 & 36 & 312 & $21 \%$ \\
\hline 20 a 24 anos & 47 & 43 & 33 & 50 & 47 & 42 & 31 & 37 & 37 & 39 & 406 & $27 \%$ \\
\hline 25 a 29 anos & 38 & 35 & 36 & 30 & 33 & 24 & 27 & 28 & 28 & 26 & 305 & $20 \%$ \\
\hline 30 a 34 anos & 16 & 14 & 26 & 12 & 15 & 20 & 14 & 13 & 12 & 24 & 166 & $11 \%$ \\
\hline 35 a 39 anos & 15 & 12 & 10 & 11 & 8 & 18 & 11 & 10 & 16 & 14 & 125 & $8 \%$ \\
\hline 40 a 44 anos & 1 & 1 & 5 & 2 & 0 & 2 & 3 & 3 & 5 & 7 & 29 & $2 \%$ \\
\hline 45 a 49 anos & 0 & 1 & 1 & 0 & 0 & 1 & 0 & 0 & 0 & 0 & 3 & $0 \%$ \\
\hline Idade ignorada & 11 & 10 & 6 & 16 & 23 & 17 & 11 & 11 & 13 & 19 & 137 & $9 \%$ \\
\hline Total & 174 & 166 & 163 & 152 & 147 & 148 & 124 & 129 & 143 & 166 & 1512 & $100 \%$ \\
\hline
\end{tabular}

Fonte: Sistema de Informação sobre Mortalidade, 2018.

Sendo assim, quando se analisa esse aspecto, percebe-se uma mudança nos parâmetros ligados ao nível de escolaridade, apontando que a maior taxa está atrelada a um maior nível educacional. Outro aspecto que deve ser levado em conta é que o nível de escolaridade materna é um indicador indireto das condições sociais e econômicas, e, consequentemente, um preditor direto do risco de mortalidade infantil (FRANÇA, 2016).

Além disso, segundo o Ministério da Saúde (2011), 35\% das mães, até 2010, no país, tinham menos de oito anos de instrução e, quando esse índice é comparado entre as regiões, demonstra que a região Sudeste apresenta $26 \%$, e Norte e Nordeste, o equivalente a $46 \%$, um número alarmante que, infelizmente, releva as desigualdades sociais do país. Quando são levados em conta os dados da pesquisa, nota-se uma redução desse índice, tornando-o mais próximo da realidade nacional.

A faixa etária (Tabela 1) das mães que os filhos evoluíram com óbito encontra-se bem variável. O número mais expressivo está na faixa de 20 a 24 anos, equivalente a $27,00 \%(n=406)$. Quando se compara esse dado com o estudo de Borba (2014), tem-se uma discordância nessa faixa etária, pois, 
em tal estudo, o número mais significativo está entre 15 a 19 anos, representando uma mudança nessa perspectiva, pelo fato de ter reduzido a incidência de mães adolescentes.

Além disso, o Ministério da Saúde (2011) retrata haver uma diminuição de gestação nos extremos de idade, principalmente a gestação na adolescência. Outro ponto é um aumento das gestações na faixa de 35 a 39, porém não muito expressivo.

Portanto, quando se avalia a escolaridade da mãe, cor e faixa etária, percebe-se uma melhoria nos parâmetros educacionais e de idade, demonstrando um maior nível de escolaridade atrelado há uma maior faixa etária de idade as mães. Além disso, quanto aos aspectos étnicos, comprova-se que $80,00 \%$ $(n=1213)$ das mães são da cor parda.

Descreve-se a seguir os resultados obtidos do cálculo do coeficiente de mortalidade infantil no município de Santarém - Pará a partir da análise de dados das tabelas do Sistema de Informação de Mortalidade (SIM) entre os anos de 2007 e 2016.

Gráfico 1- Taxa de mortalidade infantil entre o período de 2007 a 2016 no município de Santarém-PA.

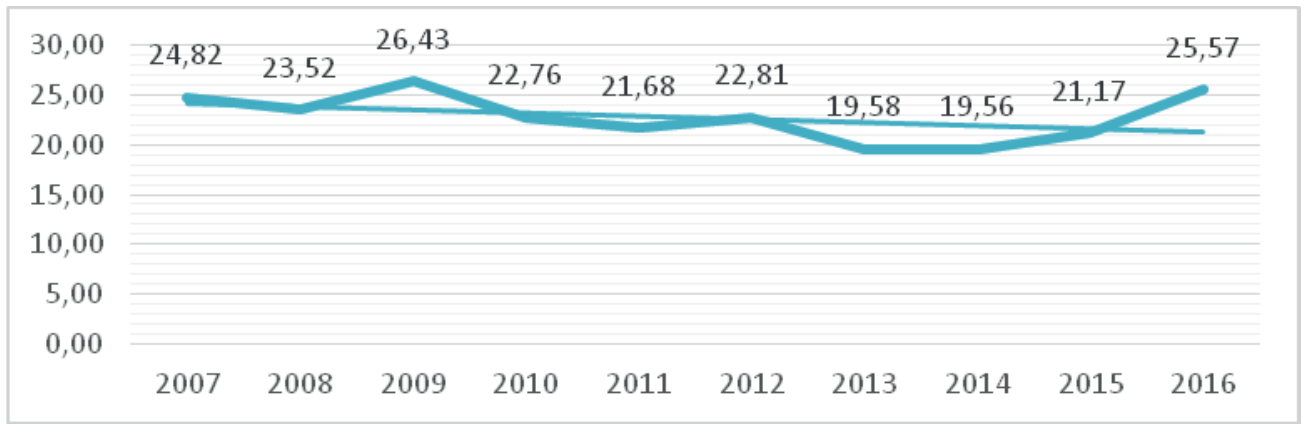

Fonte: Sistema de Informação sobre Mortalidade, 2018.

Foram notificados 1.512 óbitos infantis em Santarém-PA no período referido. Os números máximo e mínimo atingidos, levando-se em consideração a proporção de óbitos infantis para o número de nascidos vivos, foram, em 2009, de 163 óbitos para um total de 6.167 nascidos vivos, e, em 2014, de 129 para um total de 6.595 nascidos vivos, respectivamente. Ao analisar o Gráfico 1 como um todo, observa-se uma tendência de queda no índice calculado, principalmente do período de 2007, com a taxa de mortalidade infantil de 24,82, e em 2014, com a taxa de mortalidade de 19,56, que representa um decréscimo de $21,19 \%$. Observa-se, ainda, que os anos de 2015 e 2016 apresentaram um crescimento correspondente a $28,68 \%$ na taxa de mortalidade, se comparada com o valor mais baixo que ocorreu em 2014 .

Ao comparar o maior índice observado na cidade de Santarém, que ocorreu no ano de 2009, com a média nacional no mesmo período de 18,1, é possível notar que, no contexto municipal, houve uma diferença de 8,33 mortos por mil nascidos vivos a mais do que a referência nacional (IBGE, 2009). Analisando-se ainda o menor índice observado no período da pesquisa, que ocorreu em 2014, e fazendo-se a comparação com o número em nível de Brasil, cuja taxa obtida foi de 14,4, é notório que houve uma diferença menor, se comparado a 2009, entre as taxas de mortalidade infantil do município e nacional, apesar de o ano de 2014 referir a menor taxa em todo período da pesquisa, sendo essa divergência de 5,16 pontos (IBGE, 2013). 
Gráfico 2 - Taxa de mortalidade infantil entre o período de 2007 a 2016 no Brasil.

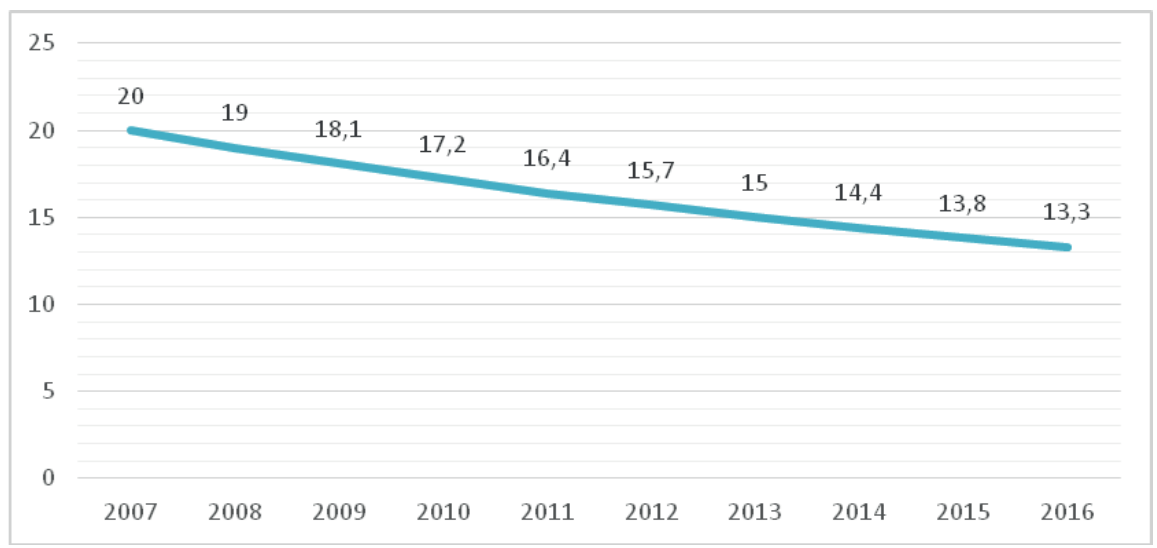

Fonte: Sistema IBGE de Recuperação Automática - SIDRA.

Observando-se o Gráfico 2, nota-se um comportamento decrescente da taxa de mortalidade infantil no país de 2007 a 2016, com maior valor em 2007, correspondendo a 20 mortos por mil nascidos vivos, e o menor em 2016, equivalente a 13,3 mortos menores de 1 ano de idade a cada mil nascidos vivos. Outro aspecto importante a ser observado diz respeito aos anos de 2015 e 2016, em que ocorreu um crescimento na taxa de mortalidade em nível municipal de 19,56 para 25,57, destoando do padrão nacional que se manteve decrescente nesses anos e mostrando a maior diferença, da taxa de mortalidade infantil Nacional, em Santarém, no ano de 2016, quando o valor foi 12,27 mortes infantis por mil nascidos vivos a mais do que o valor nacional (IBGE, 2018).

O presente estudo analisou a mortalidade infantil de acordo com a faixa etária da criança que evoluiu a óbito (Tabela 3).

Tabela 3 - Idade em dias de crianças que evoluíram com óbito de 2007 a 2016.

\begin{tabular}{|c|c|c|c|c|c|c|c|c|c|c|c|c|}
\hline IDADE/ANO & 2007 & 2008 & 2009 & 2010 & 2011 & 2012 & 2013 & 2014 & 2015 & 2016 & \multicolumn{2}{|c|}{ Total } \\
\hline 0 a 6 dias & 106 & 107 & 102 & 98 & 76 & 102 & 68 & 76 & 88 & 89 & 912 & $60 \%$ \\
\hline 7 a 27 dias & 20 & 14 & 26 & 22 & 28 & 16 & 17 & 16 & 23 & 28 & 210 & $14 \%$ \\
\hline 28 a 364 dias & 48 & 45 & 35 & 32 & 43 & 30 & 39 & 37 & 32 & 49 & 390 & $26 \%$ \\
\hline Total & 174 & 166 & 163 & 152 & 147 & 148 & 124 & 129 & 143 & 166 & 1512 & $100 \%$ \\
\hline
\end{tabular}

Fonte: Sistema de Informação sobre Mortalidade, 2018.

Tabela 4 - Proporção de crianças que evoluíram com óbito, por sexo, de 2007 a 2016.

\begin{tabular}{ccccccccccccc}
\hline SEXO/ ANO & 2007 & 2008 & 2009 & 2010 & 2011 & 2012 & 2013 & 2014 & 2015 & 2016 & Total \\
\hline MASCULINO & 103 & 102 & 97 & 85 & 87 & 89 & 67 & 81 & 76 & 90 & 877 & $58 \%$ \\
\hline FEMININO & 71 & 64 & 65 & 66 & 59 & 57 & 57 & 47 & 66 & 75 & 627 & $41 \%$ \\
\hline IGNORADO & 0 & 0 & 1 & 1 & 1 & 2 & 0 & 1 & 1 & 1 & 8 & $1 \%$ \\
\hline Total & 174 & 166 & 163 & 152 & 147 & 148 & 124 & 129 & 143 & 166 & 1512 & $100 \%$ \\
\hline
\end{tabular}

Fonte: Sistema de Informação sobre Mortalidade, 2018. 
Na proporção da mortalidade infantil por sexo, observou-se uma maior mortalidade de homens do que de mulheres, com um total de 877 óbitos do sexo masculino contra 627 do sexo feminino. Existiram, ainda, 8 mortes que foram ignoradas questões relativas ao sexo da criança.

Alguns estudos observaram um maior risco de morte em crianças do sexo masculino menores de 1 ano. Isso pode ser em decorrência do maior amadurecimento pulmonar no sexo feminino no mesmo período de vida (MENEZES et al., 1993; ARAUJO et al., 2000). Tal fato concorda com esta pesquisa, demonstrando que, do total de óbito ocorridos, $58,00 \%(n=877)$ foram do sexo masculino.

Tabela 5 - Tipo de gravidez, local da ocorrência, tipo de parto e duração da gestação de mães cujas crianças evoluíram com óbito de 2007 a 2016.

\begin{tabular}{|c|c|c|c|c|c|c|c|c|c|c|c|c|}
\hline & 2007 & 2008 & 2009 & 2010 & 2011 & 2012 & 2013 & 2014 & 2015 & 2016 & \multicolumn{2}{|c|}{ Total } \\
\hline \multicolumn{13}{|l|}{ Tipo de Gravidez } \\
\hline Única & 152 & 146 & 148 & 135 & 119 & 130 & 106 & 114 & 127 & 141 & 1318 & $87 \%$ \\
\hline Dupla & 13 & 14 & 11 & 8 & 8 & 4 & 6 & 4 & 4 & 9 & 81 & $5 \%$ \\
\hline Tripla e mais & 0 & 0 & 0 & 0 & 1 & 1 & 0 & 1 & 0 & 0 & 3 & $0 \%$ \\
\hline Ignorada & 9 & 6 & 4 & 9 & 19 & 13 & 12 & 10 & 12 & 16 & 110 & $7 \%$ \\
\hline Total & 174 & 166 & 163 & 152 & 147 & 148 & 124 & 129 & 143 & 166 & 1512 & $100 \%$ \\
\hline \multicolumn{13}{|l|}{ Local da Ocorrência } \\
\hline Hospital & 160 & 153 & 149 & 146 & 138 & 139 & 121 & 126 & 137 & 164 & 1433 & $95 \%$ \\
\hline Domicílio & 8 & 10 & 10 & 6 & 7 & 8 & 3 & 2 & 3 & 2 & 59 & $4 \%$ \\
\hline Via pública & 3 & 2 & 1 & 0 & 1 & & 0 & 1 & 0 & 0 & 8 & $1 \%$ \\
\hline Outros & 3 & 1 & 2 & 0 & 1 & 1 & 0 & 0 & 3 & 0 & 11 & $1 \%$ \\
\hline Ignorado & 0 & 0 & 1 & 0 & 0 & 0 & 0 & 0 & 0 & 0 & 1 & $0 \%$ \\
\hline Total & 174 & 166 & 163 & 152 & 147 & 148 & 124 & 129 & 143 & 166 & 1512 & $100 \%$ \\
\hline \multicolumn{13}{|l|}{ Tipo de Parto } \\
\hline Vaginal & 134 & 128 & 113 & 97 & 89 & 85 & 64 & 85 & 93 & 92 & 980 & $65 \%$ \\
\hline Cesárea & 32 & 32 & 47 & 44 & 38 & 49 & 47 & 33 & 39 & 58 & 419 & $28 \%$ \\
\hline Ignorado & 8 & 6 & 3 & 11 & 20 & 14 & 13 & 11 & 11 & 16 & 113 & $7 \%$ \\
\hline Total & 174 & 166 & 163 & 152 & 147 & 148 & 124 & 129 & 143 & 166 & 1512 & $100 \%$ \\
\hline \multicolumn{13}{|l|}{ Duração da Gestação } \\
\hline Menos de 22 semanas & 5 & 3 & 2 & 4 & 18 & 9 & 4 & 6 & 2 & 4 & 57 & $4 \%$ \\
\hline 22 a 27 semanas & 28 & 17 & 18 & 23 & 8 & 16 & 9 & 12 & 16 & 14 & 161 & $11 \%$ \\
\hline 28 a 31 semanas & 17 & 22 & 17 & 17 & 18 & 19 & 23 & 16 & 20 & 14 & 183 & $12 \%$ \\
\hline 32 a 36 semanas & 50 & 39 & 35 & 24 & 16 & 23 & 12 & 26 & 26 & 25 & 276 & $18 \%$ \\
\hline 37 a 41 semanas & 58 & 65 & 80 & 64 & 40 & 32 & 43 & 34 & 39 & 46 & 501 & $33 \%$ \\
\hline 42 semanas e mais & 6 & 9 & 4 & 6 & 1 & 2 & 2 & 4 & 1 & 2 & 37 & $2 \%$ \\
\hline Ignorado & 10 & 11 & 7 & 14 & 46 & 47 & 31 & 31 & 39 & 61 & 297 & $20 \%$ \\
\hline Total & 174 & 166 & 163 & 152 & 147 & 148 & 124 & 129 & 143 & 166 & 1512 & $100 \%$ \\
\hline
\end{tabular}

Fonte: Sistema de Informação sobre Mortalidade, 2018.

Quando de se avalia o tipo de gestação, percebe-se que $87,00 \%(n=1.318)$ é caracterizada pela primeira gestação. Esse dado entra em concordância com o estudo de Borba (2014), que afirma que $52,17 \%$ dos óbitos infantis estão relacionados com mães de primeira gestação. No entanto, não carac- 
teriza esse tipo de associação, e também, não foram encontrados estudos que relacionassem a maior mortalidade infantil com a primeira gestação.

Quanto ao local em que foi realizado o parto, nota-se que $95,00 \%(n=1433)$ foram realizados no ambiente hospitalar. Tal dado entra em concordância com o estudo de França (2016), que também constatou $97 \%$ dos partos ocorrem em hospitais com assistência médica. Porém, esse autor afirma que, no Brasil, ainda persiste uma dificuldade das mães e crianças terem acesso mais adequado ao parto por existência de grande déficit de leitos e profissionais treinados para esse tipo de atendimento, principalmente na região Norte e Nordeste do país.

Outro tipo de característica importante é o tipo parto. No estudo, verificou-se que 980 partos foram do tipo vaginal, equivalente a $65,00 \%$. Tal dado entra em concordância com Brasil (2011), que afirma ter diminuído o número de partos do tipo cesárea na última década no contexto nacional e um aumento no número de partos vaginais. No entanto, no estudo de De Paula Júnior et al. (2017), há prevalência do número de parto do tipo cesárea associado com menor tempo de duração da gestação e, consequentemente, um maior índice de mortalidade infantil. Outro aspecto relevante é a incidência do parto cesárea estar atrelado a mulheres de grupos socioeconômicos mais privilegiados, com maior escolaridade e mais frequente em mulheres brancas, conforme Brasil (2009).

A duração do período gestacional é o principal preditor para mortalidade infantil, segundo Brasil (2011), pois, quanto menor o período, tem-se um maior nível de mortalidade, devido às complicações no momento do parto e do desenvolvimento fetal. Assim, nos dados pesquisados, apresentou 501 gestações entre 37 a 41 semanas, o equivalente a 33,00\%, dentro da normalidade da idade gestacional, classificada como, a termo, segundo Zugaib (2016). Nota-se, quando se compara esses valores ao estudo de De Paula Júnior et al. (2017), realizado da cidade de Ubá, Minas Gerais, verifica-se um menor percentual de mortalidade infantil com um período gestacional menor que 37 semanas $(45,00 \%)$, inferindo-se uma redução significativa da mortalidade associada a gestações com menos de 37 semanas. Outro aspecto relevante e, de certo modo, preocupante, é o número de dados ignorados serem bem significativo $(20,00 \%)$.

Assim, quando se avalia todas as variáveis juntas, tem-se um perfil de mães de primeira gestação $(87,00 \%)$; ao local de ocorrência sendo primordialmente o ambiente hospitalar $(95,00 \%)$; ao tipo de parto, o vaginal (65,00\%); e. por último, o período de duração gestacional na faixa de 37 a 41 semanas $(33,00 \%)$.

Liderando as causas de óbitos infantis em todo o período da pesquisa estão algumas afecções originadas no período perinatal, totalizando $55,00 \%(n=834)$ dos óbitos, representando mais da metade das causas, o que corrobora com a pesquisa realizada por De Paula Junior et al. (2017), no qual se evidenciou que $82,76 \%$ dos óbitos estão relacionados às afecções originadas no período perinatal no município de Ubá-MG.

Seguido das afeç̧ões originadas no período perinatal estão malformações congênitas, deformidades e anomalias cromossômicas, com 149 óbitos, o que representa, aproximadamente, 10\% do total de óbitos no período da pesquisa. Esse dado diverge significativamente de estudo realizado por Rodrigues et al., no município de São Luiz-MA, cujos resultados mostraram que, dos 180.298 nascidos vivos, apenas $875(0,49 \%)$ apresentaram algum tipo de malformação.

Dentre as causas que menos mataram está o item Neoplasias (tumores) com apenas 4 mortes, representando 4,65\% do total de óbitos por neoplasias no estado do Pará no mesmo período analisado na pesquisa (SIM, 2018). Isso corrobora com a dissertação intitulada "Câncer da infância e da adolescência: tendência de mortalidade em menores de 20 anos no Brasil", na qual se demonstra que, para 
a faixa etária menor que um ano, foi observada tendência decrescente e constante da mortalidade por câncer no período de 1981 a 2008.

Chama atenção, ainda, o número de óbitos por doenças do aparelho geniturinário, que, em 2014, foi de 77 , equivalente a $86,5 \%$ das mortes por esse motivo de 2007 a 2016; e a categoria referente a sintomas, sinais e achados anormais em exame clínico e laboratorial que, apesar de ter sido a terceira maior causa de óbitos no período analisado, demonstrou um decréscimo significativo, passando de 23 para apenas 2 óbitos infantis em 2007 e 2016, respectivamente, ficando entre as menores causas de mortes no final do período analisado.

\section{CONCLUSÃO}

Observou-se uma mudança, quando se analisa o perfil epidemiológico das mães, abordando idade, escolaridade e cor. Havendo uma melhoria nos parâmetros educacionais e de idade, indicando um maior nível de escolaridade, há uma maior faixa etária, tornando-se, assim, mais próximo da realidade nacional. $\mathrm{E}$, quanto ao aspecto étnico, conclui-se que é mais frequente mães da cor parda, representando $82 \%$.

Quanto ao sexo, o masculino predominou sobre o feminino, com $58,00 \%$ do total. A esses resultados, podemos apontar como provável causa o maior amadurecimento pulmonar que ocorre no sexo feminino durante o período gestacional, segundo autores que trataram sobre esse tema. Ademais, com relação à idade em que houve o maior número de óbitos, constatou-se que foi no período neonatal precoce que vai de 0 a 6 dias, representando $60,00 \%$ do total.

$\mathrm{Na}$ análise quanto ao tipo de gravidez, local da ocorrência, tipo de parto e duração da gestação, tem-se um perfil de mães na primeira gestação ( $87,00 \%)$; ao local da ocorrência sendo majoritariamente o ambiente hospitalar (94,00\%); a tipo de parto, caracterizado como vaginal $(65,00 \%)$; e por último, uma idade gestacional entre 37 a 41 semanas (33,00\%).

Verificou-se que foram notificados 1.512 óbitos. A maior taxa de mortalidade infantil aconteceu no ano de 2009, com 26,43 óbitos por mil nascidos vivos, seguida por 2016, com 25,57 mortes por mil nascidos vivos.

Em relação às causas, foi importante considerar que o item "algumas afecções originadas no período perinatal", totalizando 834 óbitos, predominou sobre as outras, perfazendo $55,00 \%$ ( $n=834)$ do total, seguido por "malformações congênitas, deformidades e anomalias cromossômicas", que representam $10,00 \%$ do total de óbitos no período da pesquisa $(n=149)$ do total.

Por fim, o presente estudo se fez necessário para conhecer a realidade e as particularidades do município quanto à mortalidade infantil nos 10 anos, tendo em vista a mortalidade ser um indicador sensível de saúde tanto positivo quanto negativo. Desse modo, usar o conhecimento adquirido através do perfil epidemiológico da mortalidade infantil na cidade de Santarém-PA objetiva entender como se encontram os parâmetros de mortalidade, verificando também se entra em concordância com os dados nacionais de redução desse índice, com o intuito de implementação de políticas públicas para a região. 


\section{REFERÊNCIAS}

ARAUJO, B. F.; BOZETTI, M. C.; TANAKA, A. C. Mortalidade neonatal precoce no Município de Caxias do Sul: um estudo de coorte. J Pediatr (Rio J.) 2000; 76:2000

BORBA, G. G. et al. Fatores associados à morbimortalidade neonatal: um estudo de revisão. Saúde (Santa Maria), v. 40, n. 1, p. 9-16, 2014.

BRASIL. Ministério da Saúde. Indicadores e dados básicos Brasil, 2007. Brasília, D.F.: Ministério da Saúde, 2007. Disponível em: http://tabnet.datasus.gov.br/cgi/idb2007/matriz.htm. Acesso em: 06 dez. 2019.

BRASIL. Ministério da Saúde. Secretaria de atenção à saúde. Departamento de ações programáticas e estratégicas. Atenção à saúde do recém-nascido: guia para os profissionais de saúde. 2011.

BRASIL. Ministério da saúde. Secretaria de Atenção à Saúde. Departamento de Análise de Situação de Saúde. Manual de vigilância do óbito infantil e fetal e do comitê de prevenção do óbito infantil e fetal. 2009.

DE PAULA JÚNIOR, J. D. et al. Perfil da mortalidade neonatal no município de Ubá/MG, Brasil (20082010). Revista Brasileira de Pesquisa em Saúde/Brazilian Journal of Health Research, v. 18, n. 3, p. 24-31, 2017.

FRANÇA, Elisabeth; LANSKY, Sônia. Mortalidade infantil neonatal no Brasil: situação, tendências e perspectivas. Anais, p. 1-29, 2016.

HARTZ, Z. M. A. Avaliação dos programas de saúde: perspectivas teórico metodológicas e políticas institucionais. Ciência \& saúde coletiva, v. 4, p. 341-353, 1999.

IBGE. Projeção da População do Brasil, por Sexo e Idade, para o Período 2000/2060, Revisão 2013, e Projeção da População das Unidades da Federação, por Sexo e Idade, para o Período 2000/2030, Revisão 2013.

IBGE. (2009). censo2010. Disponivel em: https://censo2010.ibge.gov.br/noticias-censo.html?busca=1 \&idnoticia=1767\&t=2009-esperanca-de-vida-era-73-17-anos\&view=noticia. Acesso em: 17 out. 2019.

INSTITUTO BRASILEIRO DE GEOGRAFIA E ESTATÍSTICA. (2018). Disponível em: https://sidra.ibge.gov. br/tabela/3834. Acesso em: 17 out. 2019.

INSTITUTO BRASILEIRO DE GEOGRAFIA E ESTATÍSTICA .(2013). Brasil em sintese. Disponível em: https:// brasilemsintese.ibge.gov.br/populacao/taxas-de-mortalidade-infantil.html. Acesso em: 17 out. 2019.

MENEZES, A.M.; BARROS, F.C.; VICTORA, C.G.; TOMASI, E; HALPERN, R; Oliveira A. Fatores de risco para mortalidade perinatal em Pelotas, RS, 1993. Rev Saúde Pública 1998; 32:209-16.

DATASUS, 2019. Sistema de Informações sobre Mortalidade - SIM. Disponível em: https//:www2. datasus.gov.br. Acesso em 04 abr. 2019.

RODRIGUES, L. S. et al. Características das crianças nascidas com malformações congênitas no município de São Luís, Maranhão, 2002-2011. Epidemiologia e Serviços de Saúde, v. 23, p. 295-304, 2014. 
ROUQUAYROL, M.Z.; ALMEIDA FILHO, N. Epidemiologia e saúde. Rio de Janeiro. 7. ed. Medsi. 2013.

VICTORA, C. G. Intervenções para reduzir a mortalidade infantil pré-escolar e materna no Brasil. Revista Brasileira de Epidemiologia, v. 4, n. 1, p. 3-69, 2001.

ZUGAIB, Marcelo. Zugaib Obstetrícia. 3 ed. Barueri. Manole, 2016. 